\title{
Default Mode Network : Its Implications in Psychiatry
}

\author{
Dr Souvik Chakraborty \\ Assistant Professor of Psychiatry, Rampurhat Government Medical College, Rampurhat
}

The brain's "default mode network" is among the most rapidly growing neuroscientific topics of the new millennium. Since the appointment of its name in the turn of the millenium (Raichle and others 2001), the default network has garnered considerable interest for its high level of resting metabolic activity, which decreases in the face of externally-directed attention (Minoshima and others 1997; Gusnard and Raichle 2001). Though its presence was anticipated by some Neuro-scientists as early as late 1920s, it was a serendipitous discovery by a group of Neuroradiologists working on functional neuro-imaging at Washington University School of Medicine.

\section{BRIEF HISTORY OF ITS DISCOVERY}

Hans Berger, the inventor of the EEG, was the first to propose the idea that the brain is constantly busy. In a series of papers published in 1929 he showed that the electrical oscillations detected by his device do not cease even when the subject is at rest. However, his ideas were not taken seriously, and a general perception formed among neurologists that only when a focused activity is performed does the brain (or a part of the brain) become active.

1950s, Louis Sokol off, the pioneer of functional neuroimaging, and his colleagues noticed that metabolism in the brain stayed the same when a person went from a resting state to performing effortful math problems. It suggested active metabolism in the brain must also be happening during rest.

By the early 1980s PET began to receive serious attention as a potential functional neuro-imaging device in human subjects. Subtracting functional images acquired in a task state from ones acquired in a control state was a natural extension of mental chronometry. For the better part of a decade following the introduction of subtractive methodology to neuro-imaging, the vast majority of changes reported in the literature were activity increases or activations in response to tasks under investigation.

In late 1990s, Marcus Raichle \& colleagues noted that in certain circumstances subtracting task state data from control state data revealed negative responses, or task specific deactivations. Regardless of the task under investigation, the activity decreases almost always included the posterior cingulate and adjacent precuneus. They found no evidence that these cortical areas were activated at rest when compared with other areas because oxygen utilization of resting brain remained uniform despite regional variations. They concluded that the regional decreases observed commonly during task performance represented the presence of an organized functionality, that was ongoing in the resting state. This functionality was attenuated in the presence of an attentiondemanding, non self-referential task. They coined the term "Default Mode" or "Default State" of brain in their seminal paper (Raichle, 2001) to describe this functional network.

\section{ANATOMICAL LOCALIZATION}

Though Posterior Cingulate Cortex was the first area to catch the attention of the investigators, later researches showed that Default Mode Network consists of a well connected network between some anterior areas and posterior areas. Anterior part consists of Ventral and Dorsal Medial Prefrontal cortex (VMPC \& DMPC) and the posterior part consists of Posterior Cingulate Cortex (PCC) and adjacent Precuneus and Lateral Parietal Cortex, roughly corresponding to Broadman area 39. 


\section{ITS FUNCTION}

Though two decades have passed since its discovery, and number of researches on DMN sky-rocketed in last decade, its function is still not completely known. Marcus Raichle in his review article on DMN (2015) approached it by deliberating on the functions of its various subdivisions.

VMPC is a critical element in a network of areas that receive sensory information from the external world and the body via the orbital frontal cortex and convey that information to structures such as the hypothalamus, the amygdala, and the periaqueductal gray matter of the midbrain. Thus it acts as a sensory-visceromotor link concerned with social behaviour, mood control, and motivational drive, all of which are important components of an individual's personality. There have been many reports of striking personality changes and deviant social behavior appearing in premorbidly normal individuals after damage to the VMPC. The well known case of Phineas Gage is most notable among them. In studies of performance anxiety induced by task difficulty, the degree to which VMPC decreased its activity in concert with other elements of the default mode network was directly proportional to the subject's anxiety level while performing the task. With high anxiety, the VMPC decreased little if at all. As anxiety decreased with practice on the task activity so too did activity in the VMPC.

DMPC is associated with self-referential judgement. This area is also associated with theory of mind and evaluating social concepts. In an experiment by Gusnard et al. (2001) subjects were asked to make a self-referentialjudgment(i.e.,pleasantor unpleasant) about emotionally valenced pictures from the International Affective Picture System (IAPS) (Lang et al. 1997). Increases in activity were observed in the DMPC and accompanied by decreases in the VMP in subjects performing the task.

PCC along with the lateral parietal components of the default mode network, have been consistently associated with successful recollection of previously studied items. (Vincent et al. 2006). They also showed a significant relationship with the hippocampal formation.

These functional elements of the default mode network can be differentially affected during task performance by the nature of the task [e.g., presence or absence of an emotional component or an element of self-reference (Andrews - Hanna et al. 2010b, Gusnard et al. 2001)]. However, regardless of the details of a particular task, the default mode network always begins from a baseline of high activity, with small changes in this activity made to accommodate the requirements of a particular task. The available evidence indicates that the functions of the default mode network are NEVER turned off but, rather, carefully enhanced or attenuated. In a resting-state fMRI study performed on 107 juvenile offenders (Shannon et al. 2011), organizational features of their brains to their levels of impulsivity was studied. In this study, it was found that in less impulsive juveniles and normal controls, motor-planning regions were correlated with brain networks associated with spatial attention and executive control. In more impulsive juveniles, these motor-planning regions were correlated with the default mode network. The results suggested that the balance between the default mode network and the networks controlling spatial attention and executive control was critical in determining the output of cortical motor-planning areas and, ultimately the subject's level of impulsivity.

\section{ITS IMPLICATIONS IN NEURO- PSYCHIATRIC ILLNESSES}

Evidence suggests that abnormal DMN function is associated with cognitive symptoms of depression, particularly rumination. Decreased functional connectivity between the posterior cingulate cortex and the precuneus, and increased DMN dominance over the task-positive network activity, have been associated with higher levels of rumination, involving the repetition of thoughts and ideas, in depressed subject. Additionally, hyper connectivity of the subgenual cingulate cortex with the posterior cingulate cortex has been reported in 
major depressive disorder (MDD) with depressive rumination. This may be related to the function of the subgenual anterior cingulate cortex, which is implicated in channeling emotional influences from the limbic circuitry to prefrontal cortical areas. Studies reported a correlation of connectivity among the medial parietal cortex, parahippocampal gyrus, and DMN with Young Mania Rating Scale scores. Although limited data are available regarding treatment effects on the DMN in patients with mood disorders, one recent study demonstrated a normalization of DMN function in 30 percent of the patients with MDD after receiving SNRI.

Evidence for DMN dysfunction in the context of specific components of social cognitive dysfunction in Autistic Spectrum Disorder : 'self-referential processing' - the ability to process social information relative to oneself and 'theory of mind' or 'mentalizing' - the ability to infer the mental states such as beliefs, intentions, and emotions of others, is quite strong. The altered functional and structural organization of the $\mathrm{DMN}$, and its atypical developmental trajectory, are prominent neurobiological features of ASD.

Compared to healthy controls, subjects with ADHD have demonstrated stronger connections among DMN nodes than within the relevant nodes of the response inhibition network (including inferior frontal cortical, striatal, and thalamic areas). This DMN activation is thought to contribute to decreased task performance in ADHD.

Disruptions of functional connectivity between the mesial temporal lobe and the DMN have been reported in Temporal Lobe Epilepsy. These disruptions of functional connectivity in the DMN may contribute to the cognitive and/or psychiatric impairments associated with TLE.

Striatal neurons have been shown to coordinate activity not only in the basal ganglia, but also in cortical regions, specifically those in the DMN. Evidence suggests that decreased functional connectivity in the DMN may play a role in the development of Parkinson's Disease.
Decreased functional connectivity in the DMN of patients with Alzheimer Disease has been consistently demonstrated, especially between posterior (precuneus and posterior cingulate cortex) and anterior (anterior cingulate cortex and medial prefrontal cortex) regions. Changes in functional connectivity of regions within the DMN have also been found in individuals at high risk of developing $A D$, suggesting that these changes may provide potential biomarkers for AD.

Many fMRI studies have demonstrated abnormal activity within the DMN areas in patients with schizophrenia during a broad range of tasks. Abnormal functional connectivity of the DMN in schizophrenia patients has been investigated during both rest and task conditions. Bluhm et al. first reported anomalies in the temporal coherence of neuronal BOLD signals associated with the DMN in patients with schizophrenia. Specifically, patients in this study had decreased functional connectivity between the PCC seed region and other regions, including medial prefrontal, lateral parietal, and cerebellar regions, relative to controls. This report also demonstrated that positive symptoms are positively associated with functional connections between the PCC seed region and regions in auditory and attentional cortex associated with hallucinations, including bilateral temporal gyrus and premotor areas, and negative symptoms are negatively correlated with connections linking PCC to right superior temporal gyrus, ACC and premotor areas, and left inferior frontal gyrus. However, on the basis of recent evidence, functional hyper connectivity within the DMN is perhaps the most common finding in comparisons of schizophrenia patients with healthy controls. It has been extensively found in different schizophrenia populations, including chronic paranoid schizophrenia, first episode schizophrenia, individuals at ultra-high risk for psychosis, and the first-degree relatives of patients with schizophrenia. This may even be used as an endophenotype of schizophrenia. 


\section{CONCLUSION}

Though much is yet to be discovered about this large functional network, its discovery led to a paradigmatic shift in conceptualizing brain function. Its anomaly in various neurological and psychiatric illnesses is being researched in the Neuroscience laboratories across the globe. Though clinical usefulness of these findings is still elusive, we can hope that in near future it might help in better therapeutic interventions.

\section{REFERENCES}

1. Raichle ME. The Brain's Default Mode Network. Annual Review of Neuroscience. 2015; 38 : 433-447

2. Raichle, ME, MacLeod AM 2001. A default mode of brainfunction. Proc. Natl. Acad. Sci. 2001; 98 : 676-682.

3. Lee H, Lee DK, Ryu S. Default mode network connectivity is associated with long-term clinical outcome in patients with schizophrenia. Neuroimage Clin. 2019; 22 : 101-108.

4. Aarthi Padmanabhan A, Lynch CL, Menon V. The Default Mode Network in Autism Biol Psychiatry Cogn Neurosci Neuroimaging. 2017; 2 : 476-486.

5. Rachle ME, Snydera AZ A default mode of brain function : A brief history of an evolving idea NeuroImage. 2007; 37 : 1083-1090 